\title{
EFEITO DA SUPLEMENTAÇÃO COM VITAMINA A SOBRE A CONCENTRAÇÃO DE RETINOL NO COLOSTRO DE MULHERES ATENDIDAS EM UMA MATERNIDADE PÚBLICA
}

\author{
Karla Danielly da Silva Ribeiro¹, Katherine Feitosa de Araújo², Roberto Dimenstein ${ }^{3 *}$ \\ Trabalho realizado no Departamento de Bioquímica, Centro de Biociências da Universidade Federal do Rio Grande do Norte, Natal, RN
}

\author{
*Correspondência: \\ Av. Senador Salgado Filho, \\ $n^{\circ} 3000$ - Lagoa Nova \\ Natal - RN \\ CEP: $59072-970$ \\ Telefone: (84) 3215-3416 \\ ext. 212 - Fax (84) 3211-9208 \\ rdimen@uol.com.br
}

\begin{abstract}
RESUMO
Objetıvos. Avaliar o efeito da suplementação com vitamina A sobre os níveis de retinol no colostro de puérperas atendidas em maternidade pública de Natal-RN, analisando a influência do estado nutricional materno e retinol no colostro na resposta a esta suplementação.

Métodos. Foram coletados $5 \mathrm{ml}$ de sangue em jejum para análise do retinol sérico, e duas amostras de leite, antes e $24 \mathrm{~h}$ após suplementação. O consumo de vitamina A foi obtido pelo questionário de frequência de consumo. O retinol foi analisado por cromatografia líquida de alta eficiência.

Resultados. O consumo de vitamina A foi 1492,4 $\pm 1264 \mu \mathrm{gRAE} /$ dia e $23 \%$ tinha consumo provavelmente inadequado. No início (Oh) e após $24 \mathrm{~h}$ da suplementação, os grupos comparativo e suplementado apresentaram concentrações séricas de retinol de 1,3 \pm 0,4 e 1,4 \pm 0,4 $\mu \mathrm{mol} / \mathrm{L}$ ( $7 \%$ de deficiência) e 3,5 $\pm 1,7 \mu \mathrm{mol} / \mathrm{L}$ e 3,3 $\pm 1,8 \mu \mathrm{mol} / \mathrm{L}(p>0,05)$ no colostro Oh, respectivamente. No grupo suplementado o retinol aumentou no colostro $24 \mathrm{~h}$, com valores de 3,6 $\pm 1,9$ e $6,8 \pm$ $2,6 \mu \mathrm{mol} / \mathrm{L}(p<0,0001)$, respectivamente. Mulheres com níveis deficientes de retinol no colostro Oh $(<2,04 \mu \mathrm{mol} / \mathrm{L})$ transferiram mais retinol ao colostro $24 \mathrm{~h}$ do que as com níveis adequados $(326,1 \%$ e $86,5 \%$ de aumento, respectivamente).

Conclusão. A megadose foi eficaz nas primeiras $24 \mathrm{~h}$ após a suplementação. Os níveis basais de retinol no colostro influenciaram a resposta à suplementação. As parturientes que apresentaram valores baixos de retinol transferiram mais retinol oriundo da megadose ao leite do que as lactantes com níveis suficientes, possivelmente ressaltando a atuação dos mecanismos de transferência de vitamina A propostos na glândula mamária.
\end{abstract}

UNITERmos: Vitamina A. Colostro. Suplementação.

\section{INTRODUÇÃo}

A deficiência de vitamina A é um problema de saúde pública que acomete cerca de 127 milhões de crianças em idade préescolar e 7 milhões de gestantes ${ }^{1}$. Como forma de superar os fatores responsáveis por esta deficiência materna e infantil, diversos países em desenvolvimento têm efetuado medidas de intervenção visando à eliminação da carência.

A suplementação materna de vitamina A no pós-parto imediato vem sendo uma intervenção bastante utilizada em áreas de risco para deficiência de vitamina $A$ e vários estudos indicam que essa medida resulta no aumento de retinol no leite materno ${ }^{2-6}$.

Por desempenhar importantes funções nos períodos de grande proliferação celular, a vitamina A é essencial na gravidez e primeira infância, sendo transferida de dois modos da mãe para o filho: pela placenta durante a gestação e via glândula mamária (leite materno) durante a lactação, sendo esta última altamente benéfica devido aos altos níveis de retinol. Dentro dessas duas rotas, a transferência de vitamina A durante a lactação é cerca de 60 vezes maior durante os seis primeiros meses de vida do que durante toda a gestação ${ }^{7}$, uma vez que no infante as reservas hepáticas da vitamina A estão muito limitadas ao nascimento, decorrente de uma tendência à diminuição dos níveis de retinol sérico das gestantes, especialmente no último trimestre da gestação, além da existência de uma barreira seletiva placentária, a qual impede a passagem dessa vitamina para o feto ${ }^{8,9}$. Assim, em condições ideais de aleitamento, o leite materno é considerado a mais importante fonte de vitamina $A$ para multiplicar as reservas hepáticas do recém-nascido e o grande fator protetor da deficiência de vitamina $A$ até os dois anos de idade, fase de maior vulnerabilidade ${ }^{10}$.

1. Mestre - professora do Departamento de Nutrição da Universidade Potiguar - UNP e nutricionista da Secretaria Municipal de Saúde de Natal, Natal, RN

2. Graduanda do curso de Nutrição da Universidade Federal do Rio Grande do Norte - UFRN, Natal, RN

3. Médico homeopata - professor adjunto IV da Universidade Federal do Rio Grande do Norte - UFRN, Natal, RN 
Por ser o leite materno a principal fonte de vitamina A para - lactente totalmente alimentado ao seio, esta suplementação fornece o micronutriente em quantidades suficientes para formação de reserva hepática nos primeiros meses de vida ${ }^{11}$. 0 mecanismo que garante esta transferência de vitamina A materna à glândula mamária é pouco entendido em humanos.

Estudos com modelos animais afirmam que a vitamina $\mathrm{A}$ é transferida ao leite tanto ligada a proteína transportadora de retinol (RBP) quanto aos quilomícrons ${ }^{12,13}$.

Em condições de ingestão basal, cerca de $70 \%$ do retinol presente no leite é transferido à glândula mamária via RBP ${ }^{12}$. Por outro lado, a contribuição dos quilomícrons aumenta em condições de alta ingestão de vitamina A dietética ou pela suplementação, uma vez que o excesso da vitamina resulta em acréscimo de ésteres de retinil aos quilomícrons, podendo ser transferidos ao leite durante a lipólise dos triacilgliceróis nos tecidos mamários ${ }^{12,14-16}$.

Os estudos sobre suplementação de vitamina A avaliaram o impacto desta medida no estado nutricional de vitamina A materno e infantil e a influência na morbidade e mortalidade infantil, fazendo pouca referência à variação existente entre os níveis de retinol no leite das mulheres após a megadose. Sabe-se que ocorre um aumento após a suplementação, porém não foi avaliado se este aumento é semelhante em todos os casos ou se existe uma resposta diferenciada.

Portanto, este estudo avaliou o efeito da suplementação com vitamina A sobre os níveis de retinol no colostro de puérperas atendidas em maternidade pública de Natal-RN e se o estado nutricional em vitamina A e características maternas são fatores interferentes na transferência da megadose de vitamina A ao leite, uma vez que esta informação é pouco explorada na literatura.

\section{MÉtodos}

O estudo foi do tipo transversal composto por parturientes voluntárias atendidas na Maternidade Escola Januário Cicco (Natal-RN, Brasil). Entre os meses de novembro de 2005 e julho de 2006 , foram recrutadas 91 participantes para o estudo, sendo divididas em grupo comparativo (sem suplementação) e grupo suplementado (com 200.000 UI de vitamina A) para verificar o efeito da suplementação da megadose de vitamina A no leite. 0 tamanho da amostra foi calculado usando o software Statcalc (Epi-Info version 3.4.3). Considerando uma média de 200 partos por mês, para um nível de confiança de $95 \%$, estimou-se um tamanho de amostra de 47 sujeitos para o grupo teste.

Foram incluídas no estudo apenas mulheres com até 16 horas pós-parto, sem sinais de patologias, com parto a termo, concepto único sem malformação, e ausência de suplementos vitamínicos contendo vitamina A durante a gestação e no pós-parto imediato.

Após assinarem o consentimento livre e esclarecido em consonância com o Comitê de Ética em Pesquisa da Universidade Federal do Rio Grande do Norte (71433 SISNEP), as parturientes foram submetidas a formulário para coleta de dados sobre o pré-natal, parto e história clínica. Algumas informações foram transcritas do cartão de acompanhamento do pré-natal e prontuário hospitalar.

Alguns dados maternos foram obtidos para estudar sua influência na transferência de vitamina A oriunda da megadose para o leite materno. Entre eles a história reprodutiva (número de gestações anteriores), estado nutricional antropométrico materno durante o último trimestre gestacional e estado nutricional materno em vitamina $A$ (retinol no leite colostro, retinol sérico e consumo de vitamina $A$ ).

A avaliação do estado nutricional antropométrico baseou-se na coleta de medidas durante a gestação, obtendo-se o Índice de Massa Corporal (IMC) gestacional, por meio da relação de peso e altura com a idade gestacional (IG). Essas medidas foram retiradas do cartão de pré-natal, sendo consideradas as referentes a $36^{\mathrm{a}}$ semana gestacional. A classificação da adequação de IMC/ IG foi obtida através do gráfico proposto por Atalah et al. ${ }^{17}$ e as mulheres foram classificadas em baixo peso, eutrofia, sobrepeso e obesidade.

Para diagnosticar o estado nutricional em vitamina A, foram utilizados os indicadores de consumo dietético, níveis de retinol no leite e no soro das participantes.

A informação sobre o consumo dietético de vitamina $A$ foi obtida utilizando o questionário de frequência de consumo alimentar quantitativo (QFCA) correspondente ao último trimestre gestacional, previamente validado com a mesma população ${ }^{18}$. A adequação do provável consumo alimentar de vitamina A foi baseada na EAR (Estimated Average Requeriment) para gestantes adultas (550 $\mu$ gRAE/dia) ${ }^{19}$.

No período da manhã e após jejum noturno foram coletados $5 \mathrm{~mL}$ de sangue e leite materno (ordenha manual de única mama, não sugada previamente - leite Oh). A primeira ejeção do leite foi desprezada para evitar flutuações na concentração de retinol. As amostras continham entre $1 \mathrm{~mL}$ e $3 \mathrm{~mL}$ de leite. Posteriormente foi fornecida uma cápsula de retinol palmitato (200 000 UI) para o grupo suplementado. Passadas 24 horas da suplementação, uma nova coleta de leite colostro foi obtida nos dois grupos (leite 24h). No grupo comparativo, a megadose de vitamina A foi administrada após a coleta do leite $24 \mathrm{~h}$.

Para diminuir as variações de retinol em amostras casuais de leite materno, o teor de gordura do colostro foi determinado imediatamente pelo método do crematócrito descrito por Lucas et $\mathrm{al}^{20}$. As amostras foram armazenadas a $-20^{\circ} \mathrm{C}$ para posterior extração do retinol.

As alíquotas de sangue foram centrifugadas por cinco minutos (500xg) e extraídas segundo método descrito por Mayne et $a^{21}$. A concentração de retinol no soro foi expressa em $\mu \mathrm{mol} / \mathrm{L}$. A deficiência de vitamina A materna foi definida quando encontrada concentrações no soro $\leq 0,7 \mu \mathrm{mol} / \mathrm{L}(\leq 20 \mu \mathrm{g} / \mathrm{dL})^{22}$.

A concentração de retinol foi determinada por cromatografia líquida de alta eficiência (CLAE) de acordo com adaptação do método utilizado por Giuliano et $\mathrm{al}^{23}$. Etanol $95^{\circ}$ (Merck) e hidróxido de potássio a 50\% v/v (Vetec) foram utilizados para saponificação, seguida por extração com Hexano (Merck) e evaporação do extrato sob atmosfera de nitrogênio, em banhomaria a $37^{\circ} \mathrm{C}$. Em seguida, as amostras foram ressuspendidas em Etanol absoluto (Vetec) e $20 \mu \mathrm{L}$ foram aplicados no aparelho CLAE, em cromatógrafo LC-10 AD Shimadzu, acoplado a um detector SPD-10 A Shimadzu UV-VIS e integrador Chromatopac C-R6A Shimadzu com uma coluna Shim-pack CLC-ODS (M) $4,6 \mathrm{~mm} \times 25 \mathrm{~cm}$ e looping de $20 \mu \mathrm{L}$. O cromatograma evolui em eluição isocrática com fase móvel metanol, tempo de retenção de 4,3 minutos em fluxo de $1 \mathrm{~mL} / \mathrm{min}$. 0 teste de recuperação foi realizado com a adição de retinol acetato (Sigma), numa 


\begin{tabular}{|c|c|c|c|}
\hline Características & $\frac{\text { Grupo comparativo }}{(n=44)}$ & $\frac{\text { Grupo suplementado }}{(n=47)}$ & $\begin{array}{c}\text { Total } \\
(\mathrm{n}=91) \\
\end{array}$ \\
\hline Idade (anos) & $26,2 \pm 6,7^{a}$ & $24,6 \pm 4,9$ & $25,4 \pm 5,9$ \\
\hline Paridade (número de filhos) & $2,8 \pm 2,4$ & $2,0 \pm 1,1$ & $2,4 \pm 1,9$ \\
\hline \multicolumn{4}{|l|}{ Tipo de parto } \\
\hline Normal [n (\%)] & $10(23)$ & $19(40)$ & $29(32)$ \\
\hline Cesárea [n (\%)] & $34(77)$ & $28(60)$ & $62(68)$ \\
\hline Peso do recém-nascido (Kg) & $3,4 \pm 0,4$ & $3,4 \pm 0,5$ & $3,4 \pm 0,4$ \\
\hline \multicolumn{4}{|l|}{ Estado nutricional gestacional ${ }^{b}$} \\
\hline Baixo peso [n (\%)] & $5(16)$ & $8(21)$ & $13(18)$ \\
\hline Eutrofia [n (\%)] & $10(31)$ & $11(28)$ & $21(30)$ \\
\hline Sobrepeso [n (\%)] & $13(41)$ & $18(46)$ & $31(44)$ \\
\hline Obesidade [n (\%)] & $4(12)$ & $2(5)$ & $6(8)$ \\
\hline
\end{tabular}

a média \pm desvio padrão

b Estado nutricional antropométrico referente aos dados da última consulta do pré-natal (ATALAH et al, 1997). Apenas 71 mulheres tinham as informações de peso e altura registradas no cartão da gestante.

concentração de 0,06 $\mu \mathrm{mol} / \mathrm{L}$ diluído em etanol 95\% (Vetec). Foi encontrada uma recuperação de $98 \%$ do padrão e coeficiente de variação $2,7 \%$.

Os valores de retinol no colostro foram expressos em $\mu \mathrm{mol} / \mathrm{L}$ e também em $\mu \mathrm{mol} / g$ de gordura. Valores menores que $2,1 \mu \mathrm{mol} / \mathrm{L}$ para leite colostro ${ }^{24}$ e $\leq 0,3 \mu \mathrm{mol} / \mathrm{g}$ de gordura foram considerados indicativos de baixa concentração de retinol quando comparados ao leite maduro ${ }^{22}$.

Foi realizada a determinação quantitativa da Proteína $C$ Reativa (PCR) para verificar se os casos positivos de inflamação influenciavam os níveis séricos de retinol. Utilizou-se o método imunoturbidimétrico com látex (kit comercial Turbitest, Wiener lab.).

$\mathrm{Na}$ avaliação estatística foi utilizado o software Statistica 6.0. Foram utilizados o teste $T$ de Student em amostras dependentes e independentes, e Qui quadrado para variáveis categóricas. A relação entre os níveis séricos de retinol e PCR foi avaliada pela correlação de Pearson. As diferenças foram consideradas significativas quando $\mathrm{p}<0,05$.

\section{Resultados}

As características gerais das parturientes estudadas encontram-se na Tabela 1 e foram similares entre os grupos ( $p>0,05$ em todos os casos). Pode-se observar que a maioria das mulheres é multípara (mais de dois filhos), teve parto cesárea e apresentou sobrepeso (44\%) no último trimestre gestacional.

O consumo médio de vitamina A das parturientes durante a gestação foi $1492,4 \pm 1264 \mu \mathrm{gRAE} /$ dia e 23\% tinham ingestão aquém da ideal para esta fase da vida ( $<E A R=550 \mu \mathrm{g} / \mathrm{dia}$ ). Não houve diferença na ingestão de vitamina $A$ entre os grupos ( $p>0,05)$.

Os níveis séricos de retinol apresentaram valores de 1,34 $\pm 0,38 \mu \mathrm{mol} / \mathrm{L}(38,4 \pm 10,9 \mu \mathrm{g} / \mathrm{dL})$, com apenas $7 \%$ dos casos com indicação de deficiência ( $\leq 0,7 \mu \mathrm{mol} / \mathrm{L})$. Os grupos comparativo e suplementado tiveram valores de retinol sérico de 1,3 $\pm 0,4$ e $1,4 \pm 0,4 \mu \mathrm{mol} / \mathrm{L}$, respectivamente $(35,9 \pm$ $10,6$ e 40,6 $\pm 10,9 \mu \mathrm{g} / \mathrm{dL})(\mathrm{p}>0,05)$. A PCR foi analisada em 62 mulheres $(4,4 \pm 3,8 \mathrm{mg} / \mathrm{dL})$ e $90 \%$ estavam com valores

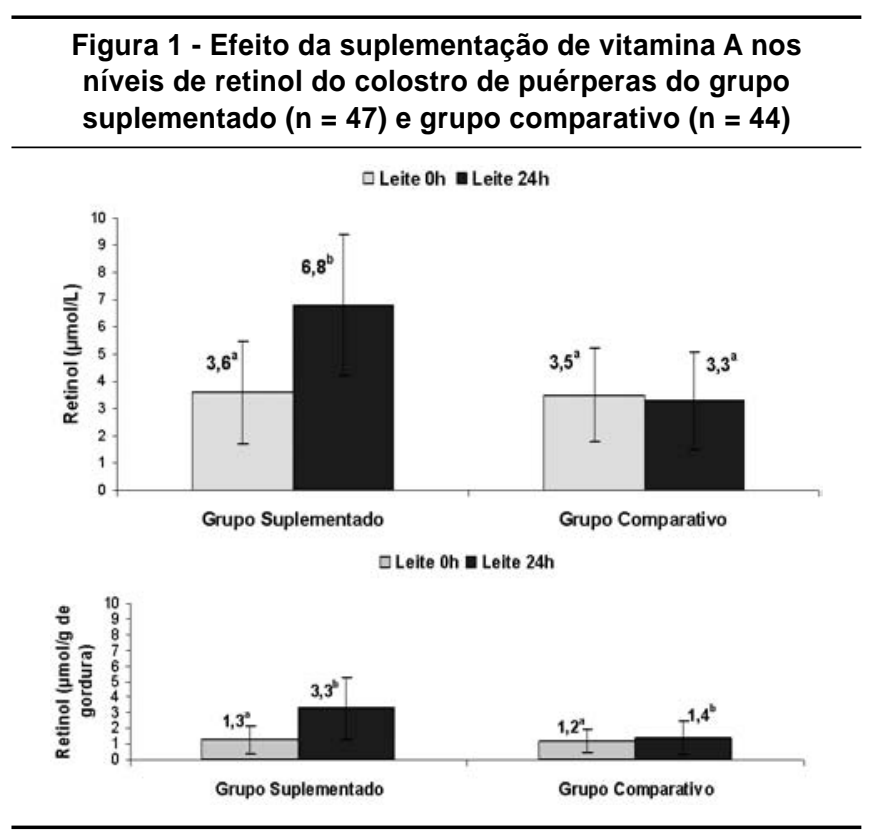

$\mathrm{A}=$ retinol expresso em $\mu \mathrm{g} / \mathrm{dL}$; ${ }^{\text {ab }}$ Diferença estatisticamente significativa entre leite Oh e $24 \mathrm{~h}$ do grupo suplementado $(p<0,0001)$ Teste t de Student para amostras dependentes; entre leite $0 \mathrm{~h}$ do grupo comparativo e $24 \mathrm{~h}$ do grupo suplementado $(p<0,0001)$; entre leite $24 \mathrm{~h}$ do grupo suplementado e $24 \mathrm{~h}$ do grupo comparativo $(p<0,0001)$ Teste $t$ de student para amostras independentes.

$\mathrm{B}=$ retinol expresso em $\mu \mathrm{g} / \mathrm{g}$ de gordura; ${ }^{\text {ab }}$ Diferença estatisticamente significativa entre leite Oh e $24 \mathrm{~h}$ do grupo suplementado $(p<0,0001)$ Teste t de Student para amostras dependentes; entre leite $0 \mathrm{~h}$ do grupo comparativo e $24 \mathrm{~h}$ do grupo suplementado $(p<0,0001)$; entre leite $24 \mathrm{~h}$ do grupo suplementado e $24 \mathrm{~h}$ do grupo comparativo $(p<0,0001)$ Teste t de student para amostras independentes.

sanguíneos altos, porém não houve influência nos níveis séricos de retinol $(p=0,69)$.

As parturientes apresentaram níveis basais adequados de retinol no colostro 3,5 $\pm 1,8 \mu \mathrm{mol} / \mathrm{L}(100,6 \pm 52,6 \mu \mathrm{g} / \mathrm{dL})$ e $22 \%$ delas foram classificadas como deficientes em relação ao leite maduro. Os grupos suplementado e comparativo tinham valores médios de retinol semelhantes no colostro $0 h(p=0,39)$ (Figura $1 \mathrm{~A}$ ). 


\begin{tabular}{|c|c|c|c|}
\hline Variáveis & $\%$ resposta & $\mathrm{n}^{\mathrm{a}}$ & $\mathrm{p}^{\mathrm{b}}$ \\
\hline \multicolumn{4}{|c|}{ Consumo dietético de vitamina A total } \\
\hline Adequado ( $\geq 550 \mu \mathrm{gRAE} / \mathrm{dia}$ ) & 146 & 35 & 0,34 \\
\hline \multicolumn{4}{|l|}{ Estado nutricional antropométrico } \\
\hline Baixo & 142,2 & 7 & \multirow{2}{*}{$>0,05^{c}$} \\
\hline Normal & 127,0 & 11 & \\
\hline \multicolumn{4}{|l|}{ Paridade } \\
\hline Multípara & 149,2 & 28 & \multirow{2}{*}{0,50} \\
\hline Primípara & 119,8 & 19 & \\
\hline \multicolumn{4}{|l|}{ Retinol soro } \\
\hline$>0,7 \mu \mathrm{mol} / \mathrm{L}$ & 140,9 & 44 & 0,54 \\
\hline \multicolumn{4}{|l|}{ Retinol leite Oh } \\
\hline
\end{tabular}

a $\mathrm{n}=$ número de amostras; b Teste $\mathrm{t}$ de student para amostras independentes; c Teste de Tukey - ANOVA.

Avaliando o efeito imediato da suplementação de vitamina A no leite materno, constatou-se que houve um aumento de retinol no leite colostro após $24 \mathrm{~h}$ da suplementação $(p=0,0001)$. 0 mesmo não aconteceu para o grupo comparativo $(p=0,29)$. Os resultados foram semelhantes quando o retinol no colostro foi expresso por grama de gordura (Figura $1 \mathrm{~B}$ ).

No grupo suplementado, foi possível observar que as parturientes apresentaram diferentes respostas à suplementação e quando estudado os possíveis fatores que poderiam interferir nesta resposta (consumo de vitamina $A$, paridade, estado nutricional antropométrico, retinol soro e retinol colostro), foi visto que esta variação estava diretamente relacionada com os níveis basais de retinol no colostro (Tabela 2 ).

\section{Discussão}

As características das mulheres estudadas foram semeIhantes às populações estudadas em Bangladesh ${ }^{3}$, Tailândia ${ }^{25}$, Espanha $^{26}$, Rio de Janeiro ${ }^{27}$. Quase metade das entrevistadas teve sobrepeso durante o último trimestre gestacional, concordando com a tendência de aumento do sobrepeso na população brasileira, principalmente entre as mulheres ${ }^{28,29}$.

Até o final da gravidez, um adequado estado nutricional com relação à ingestão de vitamina $\mathrm{A}$ e a uma dieta balanceada são importantes para garantir a transferência de nutrientes para o feto, preparando-o para o nascimento e o período de amamentação. A vitamina A cumpre um papel essencial neste período, uma vez que está intimamente envolvida em processos de grande proliferação e crescimento celular como os da gravidez, lactação e primeira infância ${ }^{30}$. O consumo médio de vitamina $A$ das parturientes durante a gestação foi adequado e cerca de $23 \%$ delas apresentaram provável inadequação dietética $(<\mathrm{EAR}=$ $550 \mu \mathrm{g} / \mathrm{dia}$ ), refletindo alta prevalência de consumo deficiente.

Neste estudo, o retinol sérico foi um dos indicadores utilizados para diagnóstico do estado de vitamina A das parturientes, sendo encontrado valores de 1,34 $\pm 0,38 \mu \mathrm{mol} / \mathrm{L}(38,4 \pm 10,9$ $\mu \mathrm{g} / \mathrm{dL}$ ) com apenas 7\% dos casos com indicação de deficiência ( $\leq$ 0,7 $\mu \mathrm{mol} / \mathrm{L}$ ). Apesar de 90\% das mulheres apresentarem PCR positiva, não houve influência nos níveis séricos de retinol $(p=0,69)$ provavelmente pela análise do soro ter sido realizada no pós-parto, situação de trauma cujo PCR eleva-se naturalmente mesmo na ausência de situações inflamatórias ${ }^{31}$.

A megadose de vitamina $A$ aumentou os níveis de retinol no colostro após $24 \mathrm{~h}$ da suplementação $(p=0,0001)$. Essa situação é vantajosa, pois os níveis séricos de retinol tendem a declinar após um mês de lactação e o colostro tem papel fundamental na formação inicial dos estoques hepáticos da vitamina $A$ do lactente $\mathrm{e}^{30,32,33}$.

Green et al. ${ }^{12}$ especulam que, durante a lactação, uma grande proporção de vitamina A oriunda de suplementos é direcionada preferivelmente a glândula mamária do que ao fígado. Esta transferência ocorre via quilomícrons (cerca de 60\%) e depende do sítio de ligação deles e da lipólise dos triacilglicerídios, por ação da lípase lipoprotéica ( $L P L)$ que é responsável pela hidrólise dos ésteres de retinil derivados dos quilomícrons.

O tecido mamário possui a enzima que esterifica o retinol $(\text { ACAT })^{34}$, sinalizando que a vitamina A transferida durante a lipólise dos lipídios dos quilomícrons pode ser reesterificada para secreção no leite ou ser estocada nas células epiteliais, fato que explica a manutenção de níveis aumentados de vitamina A no leite horas ou dias após a suplementação ${ }^{12,15}$. A confirmação desta via principal de transporte da vitamina A dietética ou de suplementos ao leite pode ser evidenciada quando concentrações de vitamina A mudam no leite após rápidas mudanças de ingestão, enquanto no sangue permanecem inalteradas ${ }^{12,13,16}$.

Observando em detalhes o percentual de aumento de retinol no colostro 24 horas após a suplementação, percebe-se uma grande variação interindividual (0 - 500\%). Na literatura não existem estudos que avaliem a variação de resposta à suplementação. Na tentativa de elucidar os fatores que poderiam interferir na distinta atuação da suplementação neste grupo, 
foi investigada a influência de algumas variáveis na resposta à suplementação (Tabela 2).

Apesar do consumo de vitamina A dietética estar relacionada aos níveis de retinol no leite, não foi encontrada esta associação. Akohoue, Green e Green ${ }^{16}$ afirmam que tal influência pode ser observada por meio de mudanças rápidas na ingestão de vitamina A, e não é detectada com consumo crônico deste nutriente.

$O$ tecido adiposo é um excelente estoque de ésteres de retinil e durante a lactação existe um aumento da sua mobilização ${ }^{35}$. Meneses e Trugo ${ }^{27}$ sugerem que a paridade pode influenciar os níveis de retinol no leite quando a lactação prévia proporciona uma alta mobilização das reservas de retinol (influenciada também pela adiposidade materna em multíparas) e alta transferência à glândula mamária. 0 estado nutricional antropométrico (que reflete a adiposidade materna em casos de sobrepeso/ obesidade) e a paridade não influenciaram na resposta à suplementação de vitamina $A$, provavelmente em virtude destes fatores exercerem influência no retinol do leite apenas em casos de ingestão basal de vitamina $A$, quando o organismo utiliza os estoques corporais.

Lourenço ${ }^{36}$ encontrou que mulheres que mantiveram ou reduziram seus níveis de retinol no leite após megadose apresentaram retinol no soro inferior ao das lactantes, que em tese se beneficiaram com a intervenção. Esse achado sugere que em casos de depleção hepática de vitamina $A$ a megadose pode ser utilizada preferencialmente pelo fígado ou por outros tecidos extra-hepáticos. Neste estudo essa relação não foi encontrada, provavelmente pelo adequado nível sérico de retinol no grupo estudado (7\% de deficiência).

Dividindo-se em dois grupos o conjunto de mães que tiveram sua resposta à suplementação avaliada, no qual o primeiro diz respeito às puérperas com retinol no colostro adequado ( $>2,09$ $\mu \mathrm{mol} / \mathrm{L}$ ) e o segundo representando as mães com níveis deficientes de retinol no colostro ( $\leq 2,09 \mu \mathrm{mol} / \mathrm{L}$ ), segundo níveis adequados no leite maduro ${ }^{24}$, foi possível verificar uma diferença significativa entre o percentual de resposta a suplementação. Mulheres com níveis deficientes de retinol no colostro transferiram mais retinol ao leite $24 \mathrm{~h}$ do que o outro grupo, encontrando um percentual de resposta equivalente a $326,1 \%$ e $86,5 \%$ de aumento, respectivamente.

Penniston, Valentine e Tanumihardjo ${ }^{11}$ encontraram evidências da existência de um limite fisiológico para a quantidade de vitamina A que pode ser transferida ao leite. Tal evento pode ocorrer pela limitação existente da transferência de vitamina A à glândula mamária via quilomícrons, provavelmente pela saturação da atividade da LPL após suplementação com vitamina $A^{37}$. Hipotetizando o fato de que mulheres com bom aporte de vitamina A no leite possuem maior atuação da LPL, provavelmente por possuírem um melhor consumo de vitamina A (não diagnosticado por inquéritos alimentares que avaliam a ingestão crônica), após suplementação a transferência de ésteres de retinol para a glândula é limitada, enquanto que no grupo com baixos níveis de retinol no colostro a LPL tem um maior espectro de atuação em relação à situação anterior até determinados níveis de saturação.

\section{Conclusão}

O uso da megadose de vitamina A no pós-parto imediato como medida de intervenção para evitar o desenvolvimento da deficiência conseguiu aumentar os níveis de retinol nas primeiras $24 \mathrm{~h}$ após a suplementação. Foi visto que esse aumento é mais efetivo em mulheres com níveis baixos de vitamina A no colostro, concordando com os mecanismos propostos para transferência da vitamina $A$ ao leite materno ${ }^{12,13,15}$.

\section{Agradecimentos}

A Maternidade Escola Januário Cicco pelo suporte oferecido neste trabalho. Ao Conselho Nacional de Desenvolvimento Científico e Tecnológico (CNPq) pelo financiamento desta pesquisa.

\section{Suporte Financeiro:}

Edital MCT CNPq / MS-DAB/SAS - N 51/2005

\section{Conflito de interesse: não há}

\section{SUMMARY}

The effect of Vitamin A supplementation UPON REtinol CONCENTRATION IN THE COLOSTRUM OF WOMEN IN A PUBLIC MATERNITY HOSPITAL

OBJECTIVE. The aim was to evaluate the effect of supplementary vitamin A upon the colostrum retinol levels in puerperal women cared for at a public maternity hospital in Natal, RN, Brazil. Analysis was conducted on the influence of the colostrum retinol and the maternal nutritional condition as response to supplementation.

METHODS. For analysis of serum retinol $5 \mathrm{ml}$ of fasting blood and two samples of milk were collected before and 24 hours after supplementation. A questionnaire was used to define the frequency of previous vitamin A intake. High Efficiency Liquid Chromatography was used to analyze the retinol.

RESULTS. Vitamin A intake was $1492.4 \pm 1264 \mu \mathrm{gRAE} /$ day and $23 \%$ probably had an inadequate intale. At the beginning of supplementation, 0 hour, and at 24 hours, the control and supplemented groups presented serum retinol concentrations of $1.3 \pm 0.4$ and $1.4 \pm 0.4 \mu \mathrm{mol} / \mathrm{L}$ (7\% deficiency) and $3.5 \pm$ $1.7 \mu \mathrm{mol} / \mathrm{L}$ and $3.3 \pm 1.8 \mu \mathrm{mol} / \mathrm{L}(p>0.05)$ at 0 hour colostrum, respectively. Retinol in the 24 hours milk of the supplemented group increased from of $3.6 \pm 1.9$ and $6.8 \pm 2.6 \mu \mathrm{mol} / \mathrm{L}$ $(p<0.0001)$, respectively. Women with deficient levels of retinol in the colostrum at 0 hour $(<2.04 \mathrm{~mol} / \mathrm{L})$ transferred more retinol to the colostrum at2 4 hours milk than those with adequate levels (an increase of $326.1 \%$ and $86.5 \%$, respectively).

CONCLUSION. The megadose of vitamin A was efficient in the first 24 hours after supplementation. The supplementation response was influenced by the basal levels of retinol in the colostrum. Parturient women with low initial levels of retinol transferred more retinol to the milk, after the megadose, than nursing women with sufficient levels. This possibly confirms the action of the Vitamin A transfer mechanisms proposed by the mammary gland. [Rev Assoc Med Bras 2009; 55(4): 452-7]

KEYwords: Vitamin A. Colostrum. Supplementation. 


\section{REFERÊNCIAS}

1. West Jr KP. Vitamin A deficiency disorders in children and women. Food Nutr Bull. 2003;24:S48-S90.

2. Bhaskaram P, Balakrishna N. Effect of administration of 200,000 UI of vitamin A to women within $24 \mathrm{hrs}$ after delivery on response to ppv administered to the newborn. Indian Pediatr. 1998;35:217-22.

3. Rice AL, Stoltzfus RJ, Francisco A, Kjolhede CL. Evaluation of serum retinol, the modified-relative-dose-response ratio, and breast-milk vitamin A as indicators of response to postpartum maternal vitamin A supplementation. Am J Clin Nutr. 2000;71:799-806.

4. Benoist B, Martines J, Goodman T. Vitamin A supplementation and the control of vitamin A deficiency: conclusions. Food Nutr Bull. 2001;22:335-7.

5. Bahl R, Bhandari N, Wahed MA, Kumar GT, Bhan MK. Vitamin a supplementation of women postpartum and of their infants at immunization alters breast milk retinol and infant vitamin A status. J Nutr. 2002;132:3243-8.

6. Stoltzfus RJ, Hakimi M, Miller KW, Rasmussen KM, Dawiesah S, Habicht JP, et al. High dose vitamin A supplementation of breast-feeding indonesian mothers: effects of the vitamin A status of mother and infant. J Nutr. 1993;123:666-75.

7. Stoltzfus RJ, Underwood BA. Breast-milkvitamin A as an indicator of the vitamin A status of women and infants. Bull World Health Organ. 1995;73:703-11.

8. Solomons NWA. Vitamin A and carotenoids. In: Bowman BA, Russel RM. Present knowledge in nutrition. $8^{\text {th }}$ ed. Washington (DC): ILSI Press; 2001. Cap. 12.

9. Azais-Braesco V, Pascal G. Vitamin A in pregnancy: requirements and safety limits. Am J Clin Nutr. 2000;71:1325S-33S.

10. Martins MC, Oliveira YP, Coutinho DC; Santos LMP. Panorama das ações de controle da deficiência de vitamina A no Brasil. Rev Nutr. 2007;20:5-18.

11. Penniston KL, Valentine AR, Tanumihardjo SA. A theoretical increased in infants hepatic vitamin $A$ is realized using a supplemented lactating sow model. J Nutr. 2003;133:1139-42.

12. Green MH, Green JB, Akohoue SA, Kelley SK. Vitamin A intake affects the contribution of chylomicrons vs. retinol-binding protein to milk vitamin $A$ in lactating rats. J Nutr. 2001;131:1279-82.

13. Ross AC, Pasatiempo AM, Green MH. Chylomicron margination, lipolysis, and vitamin $A$ uptake in the lactating rat mammary gland: implications for milk retinoid content. Exp Biol Med. 2004;229:46-55.

14. Ross AC. Cellular metabolism and activation of retinoids: roles of cellular retinoid-binding proteins. FASEB J. 1993;7:317-27.

15. Green MH, Snyder RW, Akohoue SA, Green JB. Increased rat mammary tissue vitamin $A$ associated with increased vitamin $A$ intake during lactation is maintained after lactation. J Nutr. 2001;131:1544-7.

16. Akohoue SA, Green JB, Green MH. Dietary vitamin A has both chronic and acute effects on vitamin $A$ indices in lactating rats and their offspring. J Nutr. 2006;136:128-32.

17. Atalah SE, Castillo LC, Castro SR, Aldea PA. Propuesta de um nuevo estandar de evaluación nutricional em embarazadas. Rev Med Chile. 1997;123:1429-36.

18. Nascimento THCR. Avaliação do nível de retinol e beta-caroteno no colostro humano e sua associação com o estado nutricional materno em vitamina A. Natal:Departamento de Bioquímica do Centro de Biociências, Universidade Federal do Rio Grande do Norte; 2003.

19. Institute of Medicine. Food and Nutrition Board. Dietary Reference Intake for vitamin A, vitamin K, Arsenic, boron, chromium, copper, iodine, iron, manganese, molybdenum, nickel, silicon, vanadium and zinc. Washington (DC): National Academy Press; 2001.
20. Lucas A, Gibbs J, Lyster RLJ, Baum JD. Crematocrit: simple clinical technique for estimating fat concentration and energy value of human milk. Br Med J. 1978;1:1018-20.

21. Mayne ST, Cartmel B, Silva F, Kim CS, Fallon BG, Briskin K, et al. Effect of supplemental $\beta$-caroteno on plasma concentrations of carotenoids, retinol, and $\alpha$-tocopherol in humans. Am J Clin Nutr. 1998;68: 642-7.

22. World Health Organization. Indicators for assessing vitamin A deficiency and their application for monitoring and evaluating interventions programmes: Micronutrient Series. Geneva: WHO/UNICEF; 1996.

23. Giuliano AR, Neilson EM, Kelly BE, Canfield LM. Simultaneous quantitation and separation of carotenoids and retinol in human milk by high-performance liquid chromatography. Methods Enzymol. 1992;213:391-9.

24. Macias C, Schuweigert FJ. Changes in the concentration on of carotenoids, vitamin A, alpha-tocopherol and total lipids in human milk throughout early lactation. Ann Nutr Metab. 2001;42:82-5.

25. Papanich R. Serum and breast-milk vitamin A in women during lactation in rural Chiang Mai, Thailand. Ann Trop Paediat. 2002;22:321-4.

26. Ortega RM, Andres P, Martinez RM, Lopez-Sobaler AM. Vitamin A status during the third trimester of pregnancy in Spanish women: influence on concentrations of vitamin A in breast milk. Am J Clin Nutr. 1997;66:564-8.

27. Meneses F, Trugo N. Retinol, $\beta$-caroteno, and lutein zeaxanthin in the milk of brazilian nursing women: associations with plasma concentrations and influences of maternal characteristics. Nutr Res. 2005;25:443-51.

28. Monteiro CA, Mondini L, Levy-Costa RB. Mudanças na composição e adequação nutricional da dieta familiar nas áreas metropolitanas do Brasil (1988-1996). Rev Saúde Pública. 2000;34:251-8.

29. Nascimento E, Souza SB. Avaliação da dieta de gestantes com sobrepeso Rev Nutr. 2002;15:173-9.

30. Underwood B. Maternal vitamin A status and its importance in infancy and early childhood.Am J Clin Nutr. 1994;59:517S-524S.

31. Louw JA, Werbeck A, Louw ME, Kotze TJ, Cooper R, Labadarios D. Blood vitamin concentrations during the acute-phase response. Crit Care Med. 1992;20:934-41.

32. Ross JS, Harvey P. Contribution of breastfeeding to vitamin A nutrition of infants: a simulation model. Bull World Health Organ. 2003;81:80-6.

33. Debier C, Larondelle Y. Vitamins $A$ and $E$ : metabolism, roles and transfer to offspring. Br J Nutr. 2005;93:153-74.

34. Randolph RK, Winkler KE, Ross A. Fatty acyl CoA-dependent and-independent retinol esterification by rat liver and lactating mammary gland microsomes. Arch Biochem Biophys. 1991;288:500-8.

35. OByrne SM, Wongsiriroj N, Libien J, Vogel S, Goldberg IJ, Baehr W, et al. Retinoid absorption and storage is impaired in mice lacking lecithin:retinol acyltransferase (LRAT). J Biol Chem. 2005;280:35647-57.

36. Lourenço RMS. Influência da suplementação de retinol palmitato sobre os níveis de vitamina A do leite de puérperas saudáveis. Natal: Departamento de Bioquímica do Centro de Biociências, Universidade Federal do Rio Grande do Norte; 2005.

37. Valentine AR, Tanumihardjo SA. One-time vitamin A supplementation of lactating sows enhances hepatic retinol in their offspring independent of dose size. Am J Clin Nutr. 2005;81:427-33.

Artigo recebido: 27/08/08 Aceito para publicação: 17/03/09 